\title{
The influence of malignant cell cytology screening on the survival of industrial bladder cancer cases
}

\author{
RAYMOND A. CARTWRIGHT \\ From the Yorkshire Regional Cancer Organisation, Cookridge Hospital, Leeds
}

THEODORE GADIAN

From the Clayton Aniline Company, Manchester

JOHN B. GARLAND

From the Salford Royal Hospital, Salford

SYLVIA M. BERNARD

From the Yorkshire Regional Cancer Organisation, Cookridge Hospital, Leeds

SUMmARY The influence of urinary malignant cell cytology screening has been explored in an industrial population. Survival was prolonged in the screened group partly because a greater proportion of early stage disease was being diagnosed, and partly because of other differences in the screened group which have yet to be defined.

Screening of the urine of workers exposed to the hazard of occupational tumours of the urinary tract has been carried out in the United Kingdom since 1952. A modification of the technique used by Papanicolaou ${ }^{1}$ in 1945 for early detection of cancer of the cervix is used..$^{2}$ The test is extremely accurate when carried out by a well-trained cytologist.

Malignant cell cytology (MCC) enables tumours to be diagnosed presymptomatically. The purpose of the investigation, of which this is a preliminary report was to determine whether:

(1) Tumours diagnosed by MCC are at an earlier pathological stage of development than those found after the development of symptoms.

(2) Diagnosis by MCC and consequently earlier treatment results in a higher rate of cure, or longer survival.

\section{Methods}

All male cases of bladder cancer (transitional cell carcinoma of the urothelium: ICD 8: 188 and 223.3), originating in the work force of a screening programme, were identified. Those few cases identified as a result of the initial screen were not included in this study. The group of cases used in these analyses were either diagnosed as a result of the screening test, or presented with signs or symptoms. There were no interval cases in the screened group because of the regular monthly nature of the screening test. The group who presented with signs or symptoms had not availed themselves of the screening services. For the survival analysis the date from diagnosis to death or to last follow-up was taken. In the group diagnosed by the screening procedure, survival was measured from the date when a lesion was histologically identified. This could be some weeks after the first cystoscopy was carried out subsequent to a positive urine test.

As a comparison group a retrospectively selected series of cases was used, who were treated at a hospital about four miles from the factory. The comparison group was matched by sex, age at diagnosis, and year of diagnosis. Because the hospital and factory cases were also treated by the same urologists, the two groups were matched for possible changes in treatment over the time span.

Both series have been followed up in an identical fashion and no cases were lost. The information collected included the date last seen or date of death, date of birth, date of diagnosis, the ' $T$ ' stage of the international TNM system at diagnosis, the 
occupation at diagnosis, how the diagnosis was made, and the causes of death.

Survival has been based on the log-rank technique of Peto et al. ${ }^{4}$ Other analyses were performed using the SPSS package available in the University of Leeds and the facilities offered by an ICL 1906A series computer.

\section{Results}

Eighty-eight cases from the factory were matched with a series of hospital controls. All were males. The matching was performed after assembling a random sample of hospital cases, selected within four years of the age at diagnosis of the factory cases. The mean age of the factory cases was 56.3 years, and of the hospital cases 58.4 years. No match was made for stage at presentation or the mode of diagnosis within the factory population. Of the factory cases, 33 were diagnosed as a result of the malignant cell cytology screening programme, 50 had other modes of presentation, and the remaining five did not have their mode of diagnosis recorded. Eighty-six of the hospital cases presented with symptoms or signs, and the remaining two had unknown modes of presentation.

The stage of the tumour at diagnosis varied between the three groups and is shown in Table 1. It was not possible for all cases to have the stage at diagnosis accurately ascertained, and this accounts for some omissions from the Tables. There were significant differences between the MCC group and both the other series; the MCC group had a statistically greater number of the less severe lesions.

Table 1 Stage at diagnosis

\begin{tabular}{|c|c|c|c|c|c|c|}
\hline & \multicolumn{2}{|c|}{ Factory } & \multicolumn{2}{|l|}{ Factory } & \multicolumn{2}{|l|}{ Hospital } \\
\hline & $M C C$ & $\%$ & $\begin{array}{l}\text { Signs or } \\
\text { symptoms }\end{array}$ & $\%$ & $\begin{array}{l}\text { Signs or } \\
\text { symptoms }\end{array}$ & $\%$ \\
\hline T1 & 23 & $79 \cdot 3$ & 23 & 53.5 & 45 & $52 \cdot 3$ \\
\hline $\mathrm{T} 2$ & 5 & $17 \cdot 2$ & 9 & 20.9 & 25 & 29.1 \\
\hline T3 & 1 & 3.5 & 9 & 20.9 & 15 & $17 \cdot 4$ \\
\hline T4 & 0 & - & 2 & $4 \cdot 7$ & 1 & 1.2 \\
\hline
\end{tabular}

When survival was computed by the log-rank method to include all causes of death it could be shown that the overall survival of a worker at the factory (diagnosed by either means) and a hospital case did not differ $\left(\chi^{2}\right.$ overall $\left.=0 \cdot 06, \mathrm{df}=1, \mathrm{p}=0 \cdot 81\right)$. Each group had a $50 \%$ survival at seven years, a $40 \%$ survival at 10 years, and a $25 \%$ survival at 15 years.

Differences were seen, however, when the mode of diagnosis of the factory series was taken into account.
Table 2 Observed and expected deaths from all causes by the mode of diagnosis

\begin{tabular}{lcll}
\hline & $O$ & $E$ & $O: E$ \\
\hline Factory cases & & & \\
MCC & 30 & 38.5 & 0.78 \\
Signs or symptoms & 40 & 29.5 & 1.36 \\
Hospital cases & 46 & 48.1 & 0.96 \\
\hline$\chi^{2}$ overall $=5.86 \quad 2$ df & $p=0.053$ & &
\end{tabular}

The group diagnosed by MCC survived longer, and those diagnosed symptomatically shorter, than the hospital series. The ratio of observed to expected deaths is shown in Table 2 . When all three groups were contrasted, however, the differences were just not significant at the 5\% level, although there were significant differences when the two factory series alone were contrasted $\left(\chi^{2}=6 \cdot 1, p=0 \cdot 01\right)$. The survival curve including the hospital series is shown in Fig. 1.

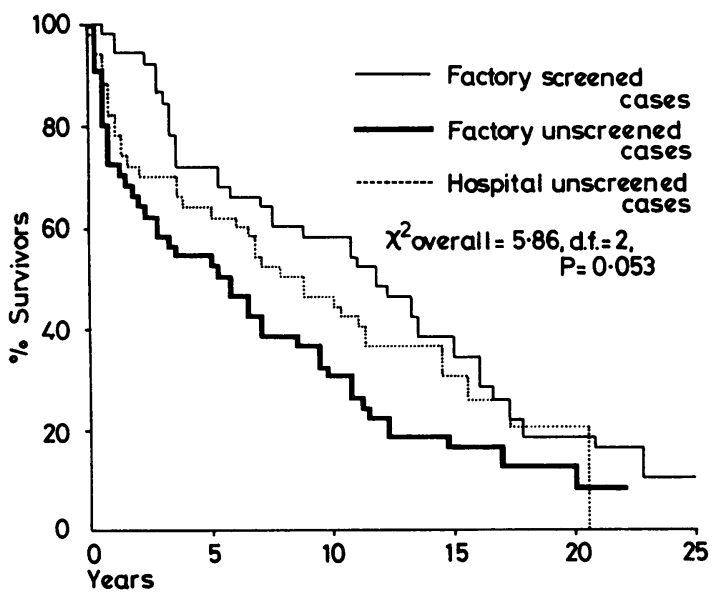

Fig. 1 Bladder cancer survival data (all causes of death included).

When account was also taken of the stage at diagnosis, the differences between the groups were reduced, as shown in Table 3 , and were no longer statistically significant $(p=0 \cdot 17)$. However, the same general pattern was found and did not completely disappear: stage at presentation accounted for less than half the differences seen.

The above data all refer to all causes of death. When the data were censored to leave deaths ascribed only to bladder cancer or the immediate complications of its treatment, the major differences between the groups were reduced to non-significant levels $\left(\chi^{2}=0 \cdot 27, p=0 \cdot 88\right)$. The survival curve is shown in Fig. 2. 


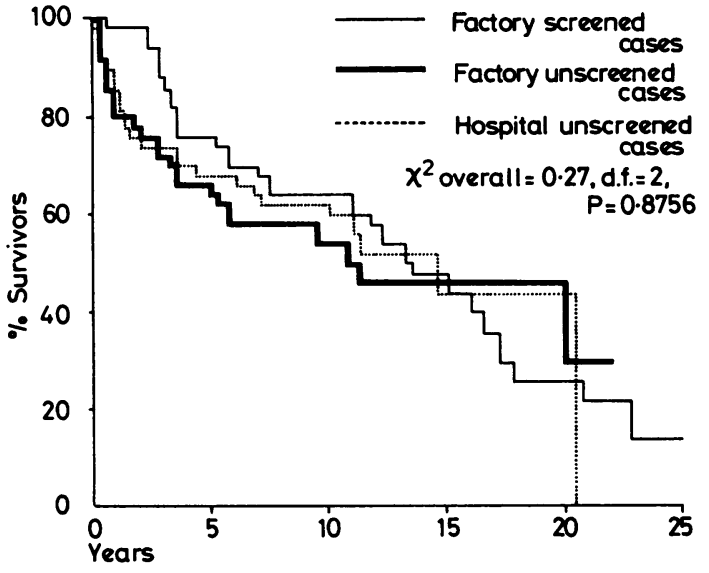

Fig. 2 Bladder cancer survival data (bladder cancer deaths only included).

Table 3 Observed and expected deaths from all causes by the mode of diagnosis with adjustment accounting for the stage at diagnosis

\begin{tabular}{llll}
\hline & $O$ & $E$ & $O: E$ \\
\hline Factory cases & & & \\
MCC & 29 & 35.6 & 0.82 \\
Signs or symptoms & 34 & 26.8 & 1.27 \\
Hospital cases & 46 & 46.6 & 0.99 \\
\hline
\end{tabular}

$x^{2}$ overall $=3.52 \quad 2$ df $p=0.17$

In Table 4 the survival timing of the MCC cases is contrasted with the rest of the factory cases for the uncensored data.

\section{Discussion}

A study of this kind is of particular relevance because the Carcinogenic Substances Regulations of 1967, which make it mandatory for urinary malignant cell cytology to be carried out in certain occupations, are under review, and there are some who believe that early diagnosis does not materially improve the prognosis in cases of bladder cancer.

Table 4 Length of time in years the screened population leads the unscreened factory group when all causes of death are considered

\begin{tabular}{llll}
\hline & \multicolumn{2}{l}{ LENGTH OF SURVIVAL IN YEARS } & \\
\cline { 2 - 4 }$\%$ & $\begin{array}{l}\text { For the screened } \\
\text { factory group }\end{array}$ & $\begin{array}{l}\text { For the unscreened } \\
\text { factory group }\end{array}$ & $\begin{array}{l}\text { Differences } \\
\text { (years) }\end{array}$ \\
\hline 70 & 5.4 & 1.5 & 3.9 \\
60 & 7.6 & 2.8 & 4.8 \\
50 & 11.7 & 5.2 & 6.5 \\
40 & 13.6 & 7.0 & 6.6 \\
\hline
\end{tabular}

Our results show that cases diagnosed by MCC tended more frequently to be stage one than the two groups presenting with signs or symptoms. As might be expected, this resulted in a better overall survival for the MCC group, in direct contrast to the factory group not diagnosed by the screening test, who did worse than both the hospital and the MCC groups. This suggests that the factory cases might have had a basically more aggressive disease. It could be postulated that some length bias would have occurred in the screened group with an over-representation of slow-growing lesions and the rapidly aggressive lesions presenting as interval cases. ${ }^{6}$ This source of bias is unlikely in this particular population, in that once self-selection had occurred in the work force, those who accepted the screening programme did so with great regularity, producing urine specimens once a month, and no interval cases arose in that group; also, cases found at the first screen were eliminated from the analyses.

Some selection bias might have occurred in that the scheme was voluntary and participation in the programme might have been influenced by past exposure. If one accepts that high exposure might lead to a particularly aggressive disease, then the screened group might have been expected to fare particularly badly: There is no evidence that this occurred and anecdotal accounts from the factory suggest that compliance with the scheme was random with respect to previous exposure and length of employment anywhere in the factory complex.

The differences in overall survival were reduced when the stage at diagnosis was taken into consideration, supporting the concept that stage differences accounted in part for the improved overall survival of the MCC group. The improved survival time for this uncensored data for the MCC cases versus the rest of the factory cases is shown in Table 4. Much of the improved survival of the screened group was due to the excess of the stage one cases found by the screening programme. This is a lead time bias. Adjustments for stage in the survival statistics reduced the $\chi^{2}$ value from 5.86 to 3.52 .

There are several other problems with these analyses. Some of the biases are compensated for by the fact that the urologists were the same in the hospital and the factory and matches have been made for year of treatment. Nevertheless, the mean ages of patients in the two groups, hospital and factory, were not exactly matched and this could have influenced the survivals marginally. The question as to whether or not to use censored or uncensored data is not at first sight clear in this study. Censoring the data means that only bladder cancer deaths are considered in the computations. Some loss of sensitivity occurs when all causes of death are considered, because in 
25 years of follow-up many 'natural deaths' due to causes other than bladder cancer will occur. This is balanced and probably outweighed by the bias introduced by the censoring. This bias is due to the inability to separate deaths due to bladder cancer from those arising from other causes. It is likely to be large in this study for a variety of reasons.

The results of censoring show that causes of death other than bladder cancer are proportionately greater in two of the three subsets. This could be fortuitous. The accuracy of death certification is unassessable in this study, spanning many years, and great suspicion can be attached to a number of certificates which attribute the cause of death to lung or brain malignancies without mention of bladder cancer; these could be secondary tumours, for example. Other studies have shown that a significant error can exist. ${ }^{7}$ There are thus strong arguments for examining only the uncensored results. Both modes of analysis, however, are presented in this report for completeness.

Questions like these are being explored further in an attempt to elucidate what is meant by a 'cure' in these circumstances, and what might be expected from this type of population irrespective of its bladder cancer. The marginal statistical significance of some of these results makes it appropriate to suggest that additional numbers added to the present series would be of great value, although the contrast between the factory cases alone, screened and unscreened, is highly significant.

There has been a tendency to reject many tests of screening, often because clearly defined study populations were not available. There was a fear that this would also be the case with urinary malignant cell cytology. However, our findings indicate that the screened population initially survives longer than the hospital or the rest of the factory population. The differences in survival amount to years, contrasting the MCC group with the unscreened factory group. These results are not just statistically significant when the hospital cases are included. Nevertheless, the trend in all types of survival analysis is for the MCC screened group to survive longer than the other groups and, for this reason, the consistent lead of the screened group is taken to be a real rather than a fortuitous phenomenon.
We thank Dr. J. Cuzick of the ICRF Cancer Epidemiology and Clinical Trials Unit at Oxford for his advice and interest. We also thank the other consultants at Salford Royal Hospital and the management of Clayton Aniline for their help, in particular Mr. J. Bevan, personnel director, who has given us every encouragement and assistance, and Mr. D. Levick, whose knowledge and expertise about the work force expedited the study. We also acknowledge the contribution made to the present study by Professor T. S. Scott's original work.

The work was financed and carried out under the auspices of the Yorkshire Regional Cancer Organisation, Occupational Cancer Group.

Reprints from Dr. R. A. Cartwright, Yorkshire Regional Cancer Organisation, Cookridge Hospital, Leeds LS16 6QB.

\section{References}

${ }^{1}$ Papanicolaou GN, Marshall VF. Urine sediment smears as a diagnostic procedure in cancers of the urinary tract. Science 1945; 101: 519-20.

${ }^{2}$ Crabbe JGS. Exfoliative cytological control in occupational cancer of the bladder. $\mathrm{Br} \mathrm{Med} J \mathrm{1952}$; ii: 1072-6.

${ }^{3}$ Crabbe JGS, Cresdee WC, Scott TS, Williams MHC. The cytological diagnosis of bladder tumours amongst dyestuff workers. $\mathrm{Br} J$ Ind Med 1956; 13: 270-6.

${ }^{4}$ Peto R, Pike MC, Armitage Pet al. Design and analysis of randomized clinical trials requiring prolonged observation of each patient. I: Introduction and design. Br J Cancer 1976; 34: 585-612.

${ }^{5}$ Peto R, Pike MC, Armitage P et al. Design and analysis of randomized clinical trials requiring prolonged observation of each patient. II: Analysis and examples. Br J Cancer 1977; 35: 1-39.

${ }^{-}$Cole $\mathrm{P}$, Morrison AS. Basic issues in population screening for cancer. J Natl Cancer Inst 1980; 64: 1263-72.

${ }^{7}$ Davies JM. Two aspects of the epidemiology of bladder cancer in England and Wales. Proc R Soc Med 1977; 70: 411-3. 